\title{
Jóvenes y precariedad laboral: trayectorias laborales por los márgenes del empleo
}

\author{
Elsa Santamaría López \\ Área de Psicología y Ciencias de la Educación, Universitat Oberta de Catalunya \\ <esantamarial@uoc.edu>
}

\begin{abstract}
Gaur egungo gazteek bizi dituzten ezegonkortasun eta lana lortzeko zailtasunek egiturazko izaera hartu dute laneko merkatuan. Artikulu honetan fenomeno hori berrikusten da $\mathbf{3 0}$ urte inguru dituzten gazte helduen laneko ibilbideak aztertuz. Betiere 'enpleguaren ertzeetan' dauden gazte horiek unibertsitateko prestakuntza osatu ostean, bekak, praktika-kontratuak, aldi baterakoak edo kontraturik gabeko lanak bete dituzte. Sakonean egindako elkarrizketa batzuetan jasotako euren esperientzia oinarri hartuz, gogoeta burutzen da prestakuntza, enplegua eta ezegonkortasunaren inguruan azaltzen diren laneko ibilbideetako beste dimentsio batzuen arabera. Halaber, egungo enplegua lortzeko paradigmaren baitan enplegagarritasunaren gaiak aztertzen dira, izan ere, gero eta gehiago barneratzen dute hori gazteek berauek, eta logika horrek garrantzia nabarmena du egungo enpleguaren inguruan sortzen diren subjektibitateak osatzeko garaian.
\end{abstract}

\section{HITZ-GAKOAK:}

laneko ezegonkortasuna, gazte helduak, laneko ibilbideak, gizarte- eta lan-politika, subjektibitateak.
La precariedad y las dificultades de acceso al empleo que sufren las personas jóvenes son cuestiones que han adquirido un carácter estructural en el mercado de trabajo. Este artículo revisa este fenómeno a partir del análisis de trayectorias laborales de jóvenes adultos, de alrededor de los 30 años, en los 'márgenes del empleo', jóvenes que, tras formarse en la universidad, han pasado por sucesivas becas, contratos en prácticas, contratos temporales 0 trabajos sin contrato. A partir de sus experiencias, recogidas en entrevistas en profundidad, se reflexiona sobre el desajuste entre la formación y el empleo, y sobre otras dimensiones de lo precario que aparecen en sus trayectorias laborales. Asimismo, se abordan cuestiones que tienen que ver con la empleabilidad, como el actual paradigma de las políticas de acceso al empleo, cada vez más interiorizado por parte de las personas jóvenes y cuya lógica juega un papel importante en las subjetividades que actualmente se crean en torno al empleo.

\section{Palabras ClaVe:}

precariedad laboral, jóvenes adultos, trayectorias laborales, políticas sociolaborales, subjetividades. 


\section{Introducción: la débil situación de las personas jóvenes en el mercado de trabajo}

Las condiciones laborales del mercado de trabajo español de las últimas décadas han generado un escenario precario de lo sociolaboral que funciona como sustrato de la nueva economía de mercado. Esas condiciones laborales más precarias se han agudizado con la crisis que vivimos desde finales de 2008 , que ha venido a teñir de más oscuro el escenario laboral, generando más desempleo y reduciendo las oportunidades de empleo, incluso del empleo precario.

Según los datos de la Encuesta de Población Activa del primer trimestre de 2012, en el Estado español la destrucción de empleo, junto con la caída en el número de activos, ha provocado que el número de parados aumentase, situando la tasa de paro en el $24,44 \%$. Pero son precisamente las personas jóvenes uno de los colectivos a los que más está afectando la destrucción de empleo en esta crisis económica. La tasa de paro de las personas menores de 34 años se ha triplicado desde que comenzó la crisis, al pasar del $10,8 \%$ del tercer trimestre de 2007 al $33 \%$ en el primer trimestre de este año. Esto significa que, actualmente, uno de cada tres jóvenes menores de 34 años que quiere y puede trabajar está en el paro.

Como ya sucedió en crisis precedentes, la gravedad del desempleo se intensifica en las personas más jóvenes, en las menores de 25 años, para quienes durante esta crisis la tasa de paro ha llegado a máximos históricos, alcanzando a uno de cada dos jóvenes durante 2012. Además sus oportunidades de empleo se han visto limitadas, ya que, según la misma fuente, casi la mitad de las personas jóvenes menores de 34 años lleva más de un año buscando trabajo, proporción que antes de comenzar la crisis apenas llegaba al $15 \%$.

La situación en el País Vasco está menos deteriorada tanto para las personas jóvenes como para el resto de la población trabajadora. Ésta es la comunidad autónoma con menor tasa de paro (13,6\%) y también con menor número de personas jóvenes menores de 34 años desempleadas; no obstante, el desempleo alcanza a un $20,9 \%$ de este grupo de edad -porcentaje que se asemeja más a la realidad del resto de países europeos-.

Si bien los datos muestran una realidad laboral bastante oscura para las personas jóvenes, y para las no tan jóvenes, esta situación no es nueva, ni se debe únicamente a la crisis económica que vivimos en los últimos años. Como decíamos al principio, la evolución de las condiciones laborales de las últimas décadas han mantenido a las personas jóvenes en una situación de clara desventaja respecto al resto de la población trabajadora. La temporalidad de sus contratos laborales y la precariedad de sus condiciones laborales se convirtieron en la norma en la década precedente a la actual crisis económica, pero como se trataba de un periodo de bonanza económica, apenas se tuvieron en cuenta los graves efectos que estaban provocando.
Ahora, con la crisis, esa vulnerabilidad en los empleos de las personas jóvenes ha sido determinante en la destrucción de empleo juvenil. De este modo, la crisis ha hecho más evidentes sus condiciones laborales de precariedad y la desigual distribución de la vulnerabilidad entre las personas trabajadoras, mermando las pocas posibilidades de mejora que antes se podían atisbar y, al mismo tiempo, ha puesto de relieve la escasez de mecanismos políticos para revertir esta situación. Esta triple conjunción de precariedad prolongada en el tiempo, destrucción de empleo y de expectativas laborales producto de la crisis económica, y falta de respuestas políticas es determinante a la hora de analizar las causas de lo que está provocando la indignación no sólo de jóvenes, sino de gran parte de la población.

Para conocer el trasfondo de la actual situación y comprender que las circunstancias que sufren ahora las personas jóvenes en el mercado laboral viene de lejos, nos vamos a remitir a un estudio ${ }^{1}$, realizado en las puertas de la crisis, de trayectorias de jóvenes en los 'márgenes del empleo', esto es, con trabajos desprotegidos y desregulados y que difícilmente llegan a la categoría de empleos. Se trata de un estudio sociológico de corte cualitativo ${ }^{2}$ que pretendía fijarse en cómo la tendencia hacia la precarización del empleo estaba repercutiendo en los itinerarios laborales de las personas jóvenes, y no precisamente en las que menor formación tenían, sino en aquellas que, pese a haber completado sus estudios universitarios, estaban teniendo, contra todo pronóstico, serias dificultades de acceso al empleo.

El contenido del artículo se estructura del siguiente modo. En un primer apartado, se abordan los principales cambios acontecidos en las trayectorias laborales de la juventud y se presenta una tipología de trayectorias laborales marcadas por el sello de la incertidumbre y la precariedad. En el segundo apartado, se exponen algunos de los efectos de la precarización del empleo en la relación que establecen las personas jóvenes con el empleo. En el tercer apartado, se prestará atención a las políticas de empleo dirigidas a las personas jóvenes y, en especial, a las consecuencias de la lógica de la empleabilidad. Y se cierra el artículo aportando una breve reflexión sobre el proceso de convertirse en trabajador/a actualmente, esto es, sobre la emergencia de nuevas subjetividades relativas al empleo desde las que se manifiestan las paradojas del contexto de precarización laboral y de las nuevas dinámicas institucionales en las que se desarrollan.

Se trata de un trabajo de tesis doctoral realizado por la autora, dirigido por Alfonso Pérez-Agote y defendido en la Universidad del País Vasco en octubre de 2009. Una versión abreviada se encuentra en Santamaría, 2011.

2 El trabajo de campo comprende grupos de discusión (10), observación participante (8) y entrevistas en profundidad (17); y son estas últimas a las que se hace referencia en este artículo. 


\section{Transitando los márgenes del empleo: la fragmentación de trayectorias laborales}

En el contexto español, estudios interesados en los procesos de inserción laboral juvenil (García Blanco y Gutiérrez, 1996; Martín Criado, 1998; García Espejo, 1998; García-Montalvo y Peiró, 2001; Martínez Martín, 2000 y 2003; Santos Ortega, 2003; Davia, 2004; Casal, 1996; Casal et. al., 2006), vienen insistiendo desde hace tiempo en la desestandarización de los itinerarios de inserción laboral y en la diversificación de tipos de trayectorias laborales de la juventud. Podemos resumir los principales cambios acontecidos a partir de las siguientes características:

- Ha desaparecido la linealidad con que la se producían los acontecimientos vitales, y, con ella, la previsibilidad. Ahora, en los itinerarios, ciertas etapas se prolongan (la de los estudios, la de formación en sucesivos contratos de prácticas y becas, la de permanencia en casa de los padres) y ciertos movimientos se vuelven reversibles (dejar el trabajo y retomar los estudios, ir al paro para iniciar otra actividad) [European Group for Integrated Social Research, 2001; Du Bois-Reymond y López Blasco, 2004; Walther, 2006, Machado País, 2007]. Esto está provocando la superposición de roles (estudiantes trabajando, trabajadores estudiando, desempleados que están muy activos, jóvenes independientes viviendo en casa de los padres, jóvenes dependientes viviendo fuera de casa de sus padres).

- El conjunto de transiciones (laboral/económica/ familiar) que solían producirse paralelamente de forma consecutiva en las trayectorias vitales juveniles se presenta hoy de forma fragmentada. Por ejemplo, la transición de los estudios al empleo que se produce en la etapa joven ha sufrido tantos cambios que ha perdido su significado tradicional. Los mecanismos tradicionales de acceso al empleo son necesarios, pero no suficientes para acceder a él. En las actuales condiciones de empleo, a un currículo extenso, una formación específica y actualizada, y a una disposición certera para el empleo hay que añadir más elementos continuamente. Sobre todo, se trata de añadir capacidades, habilidades, actitudes y competencias que se adecuen al desempeño de las nuevas tareas y a las nuevas formas de organización del mercado laboral.

- Las trayectorias laborales tradicionales en las que se reconocían ciertos colectivos han perdido su carácter representativo a favor de trayectorias cada vez más individualizadas (Walther, 2006). En este sentido, se afirma que no hay modelos colectivos que seguir y, por lo tanto, las trayectorias laborales se viven cada vez más como caminos 'personales e intransferibles'.

Ante este panorama, el estudio de las trayectorias sociolaborales se convierte en un campo de análisis ideal para comprender las tensiones y paradojas con las que cotidianamente se enfrentan las personas en su relación con el empleo. Tal es el caso de las trayectorias analizadas en este estudio, que tienen como protagonistas a jóvenes que se han formado en la universidad, han crecido en la flexibilidad económica y en la temporalidad del empleo -a la par de su regulación-, y sólo conocen de oídas, por lo que les contaron, lo que fue la estabilidad en el empleo.

Tras su formación universitaria, sólo han encontrado trabajo temporales y precarios, y por eso decimos que han ido transitando los márgenes del empleo, porque sus situaciones laborales se mueven en un continuum entre el no empleo y los cuasi empleos (trabajos que no llegan a ser empleos). En ese continuum, se pueden reconocer trabajos informales, aquellos que se realizan en negro y en los sectores de la economía sumergida, pero también otros regulados, como son los contratos de prácticas, las becas, los contratos eventuales, los empleos subvencionados y ciertos trabajos voluntarios, entre otros.

En el lenguaje oficial de las estadísticas laborales - como la Encuesta de Población Activa, la Encuesta de Coyuntura Laboral y los registros de la Seguridad Social-, estas situaciones aparecen y desaparecen continuamente, en algunos casos resultan complejas de contabilizar (situaciones esporádicas de empleo), y en la mayoría, simplemente, no se registran (por ejemplo, el trabajo voluntario o las becas).

La peculiaridad de sus trayectorias es que han cumplido los requisitos necesarios para encontrarse en empleos más protegidos, pero no es así: se encuentran transitando los márgenes del empleo. Su formación y cualificación universitaria les debería haber colocado en posiciones laborales en las que empezar una carrera profesional, pero para la mayoría no está siendo así, y andan encadenando empleos, muchos de los cuales apenas tienen relación entre sí. Además, su edad, en torno a los 30 años, les está avisando de que ya deberían haber dejado atrás la fase socialmente reconocida para el empleo juvenil, que coincide, precisamente, con ese empleo al margen, repleto de trabajillos, chapuzas, curros y trabajos alimenticios.

Pese a que sus condiciones de partida son similares (provienen de clases medias y trabajadoras, han alcanzado un nivel formativo superior y su edad ronda los 30 años), sus trayectorias laborales en la precariedad del empleo, podemos afirmar que, aunque con rasgos comunes, no son similares. Los resultados de esta investigación revelan una mayor heterogeneidad de recorridos de la juventud en el contexto de precariedad laboral que la habitualmente reconocida por la mayoría de los estudios académicos sobre este tema.

A partir del análisis de los relatos, extraídos de entrevistas en profundidad a jóvenes con las anteriores características, podemos reconocer, al menos, tres tipos trayectorias: flexibles, precarias y precarizadas. Estas trayectorias por los márgenes del empleo se representan en la zona gris del Cuadro 1 , y son el resultado de la extensión de las condiciones preca- 
rias de empleo. Por eso, cuanto más se extiende esa zona gris, más se estrecha la zona correspondiente a la protección y la estabilidad laboral, donde son posibles las trayectorias estables y ordenadas, cada vez más minoritarias.

Esta tipología de trayectorias no responde exactamente a una fragmentación gradual, sino a un desplazamiento por dos ejes. Un eje que tiene que ver con las condiciones laborales que van del polo de la protección y la seguridad en el empleo (estabilidad) al de la precarización del empleo (inestabilidad); y otro eje, que tiene que ver con la dimensión temporal, con la continuidad o la discontinuidad de determinadas condiciones laborales.

\begin{tabular}{|c|c|c|}
\hline \multicolumn{3}{|c|}{$\begin{array}{l}\text { Cuadro 1. Procesos de fragmentación de las trayectorias } \\
\text { sociolaborales }\end{array}$} \\
\hline Condiciones laborales & $\begin{array}{l}\text { Dimensión } \\
\text { temporal }\end{array}$ & Trayectorias \\
\hline \multirow{2}{*}{$\begin{array}{l}\text { Protección laboral } \\
\text { (estabilidad y } \\
\text { regulación) }\end{array}$} & $\begin{array}{l}\text { Estabilidad } \\
\text { continua }\end{array}$ & $\begin{array}{l}\text { Trayectorias } \\
\text { ordenadas }\end{array}$ \\
\hline & $\begin{array}{l}\text { Estabilidad } \\
\text { discontinua }\end{array}$ & $\begin{array}{l}\text { Trayectorias } \\
\text { flexibles }\end{array}$ \\
\hline \multirow{2}{*}{$\begin{array}{l}\text { Precariedad laboral } \\
\text { (inestabilidad y } \\
\text { desprotección) }\end{array}$} & $\begin{array}{l}\text { Inestabilidad } \\
\text { discontinua }\end{array}$ & $\begin{array}{l}\text { Trayectorias } \\
\text { precarias }\end{array}$ \\
\hline & $\begin{array}{l}\text { Inestabilidad } \\
\text { continua }\end{array}$ & $\begin{array}{l}\text { Trayectorias } \\
\text { precarizadas }\end{array}$ \\
\hline
\end{tabular}

Fuente: Elaboración propia.

Las primeras trayectorias de la zona gris son las trayectorias flexibles, donde puede darse una cierta estabilidad, por ejemplo, respecto al sector o la profesión en que se trabaja, aunque deba hacerse con contratos de trabajo discontinuos. Se trata de trayectorias que disfrutan de una cierta estabilidad, aunque ésta deba ser renovada cada cierto tiempo. Con la prolongación en la temporalidad y el estancamiento en cierta inseguridad, la percepción de la precariedad laboral y las expectativas en cuanto al empleo van cambiando. En estas trayectorias, se busca el alejamiento de la precariedad laboral y el ascenso de estatus socioeconómico, y es como si 'se viera una luz al final del túnel'.

Las trayectorias precarias son aquellas de quienes, ante la lejanía de un empleo y estatus laboral de calidad, buscarán apañárselas en el terreno inestable del empleo. Cambiar de empleo, pasar periodos de desempleo, entrar y salir consecutivamente del mercado laboral, buscar trabajo en otro país, formarse en otro campo laboral, cambiar de profesión, autoemplearse: es la movilidad laboral que se experimenta en estas trayectorias, llenas de claroscuros. Las desconexiones $\mathrm{y}$, a veces, conexiones forzadas entre experiencias laborales producto de los procesos de precarización del empleo, dibujan trayectorias sociolaborales con 'accidentes' demasiado frecuentes. La posibilidad de accidentes hace que las trayectorias laborales se vean como inciertas: en ellas caben las casualidades y el azar, así como los infortunios y también los golpes de suerte.
El último tipo de trayectorias que destacamos, las trayectorias precarizadas, son aquellas en las que la precariedad y la inestabilidad laboral se convierten en una constante. Las posibilidades de mejora tienden a desaparecer cuando se produce un estancamiento de las condiciones laborales precarias y se agudizan sus consecuencias. Las personas jóvenes inmersas en estas trayectorias intentarán evitar la caída en un paro prolongado, porque no sólo les aleja de la actividad laboral, también de sus proyecciones profesionales y ello les situaría en una clara situación de exclusión social.

Las causas estructurales de la precarización del empleo son palpables en los tres tipos de trayectorias, pero en estas últimas son mucho más evidentes, ya que ponen en relieve los problemas de regulación de los mercados de trabajo y las abusivas prácticas empresariales de selección y de gestión de personal, dejando un escaso margen de maniobra a sus protagonistas. Este hecho es lo que diferencia las trayectorias precarias de las precarizadas, esto es, que en las precarizadas sus protagonistas no se reconocen a sí mismos como sujetos activos de sus itinerarios laborales y, por extensión, vitales.

\section{Implicaciones de una socialización en el empleo precario}

Como hemos podido comprobar en este estudio, los modelos de socialización laboral vigentes son contradictorios. Funcionan todavía con unos parámetros que en un tiempo pasado pudieron funcionar, pero que ahora no son válidos. Además, se producen en una etapa, la de la juventud, que también se está transformando, y cuya especificidad resulta cada vez es más difícil de establecer.

No podemos determinar el alcance de las trayectorias sociolaborales estudiadas, si seguirán ancladas en la temporalidad de los empleos, esto es, si se estabilizarán en la "trampa" que supone la temporalidad contractual (Toharia y Cebrián, 2007), o si mejorarán paulatinamente sus condiciones laborales, ocupando posiciones laborales más estables, como si de un “puente" hacia la estabilidad se tratara (Gash, 2008). En todo caso, para algunas trayectorias, la temporalidad del empleo será una experiencia que abarque toda la vida laboral. La temporalidad laboral está siendo un rasgo que define el modelo de empleo español y que ha llegado a afectar a más de un tercio de la población, independientemente de su edad. Y aunque la temporalidad tiende a descender con el aumento de la edad, seguirá habiendo un segmento de personas cuya experiencia juvenil en el terreno laboral no será diferente de su experiencia adulta³.

3 Son pocos los análisis cuantitativos realizados que midan los efectos de la precariedad del empleo en las trayectorias laborales, y se debe principalmente a la escasa longitud de los datos disponibles. Véase, por ejemplo, Verd y López Andreu (2012). 
De aquí podemos obtener al menos tres consideraciones. En primer lugar, el contexto laboral propicia la formación de trayectorias sociolaborales como las estudiadas, desde las flexibles a las más precarizadas, cuyo destino parece ser la movilidad entre diferentes empleos: vidas laborales en un continuo movimiento entre empleos, bien para progresar laboralmente, bien para subsistir. En segundo lugar, resulta entonces poco adecuado reducir el problema de la temporalidad y de la precariedad laboral a una cuestión juvenil, más bien debe plantearse como un problema estructural de nuestro modelo de empleo. En tercer lugar, lo que podemos constatar, independientemente de hacia dónde vayan estas trayectorias, son los efectos de una socialización laboral anclada en la experiencia de la precariedad. No podemos llegar a delimitar su alcance, pero sí confirmar que la experiencia de la precariedad laboral está moldeando procesos sociales como el acceso al empleo, la transición a la vida adulta y las formaciones familiares, y ciertos proyectos y expectativas de futuro que están dotando de significados diferentes tanto al trabajo como al empleo.

A modo de balance del trabajo de campo realizado, vamos a señalar algunos de los efectos de este tipo de socialización laboral, plenamente conscientes de que las particularidades de las personas entrevistadas no son extrapolables al conjunto de jóvenes adultos, sobre todo respecto a sus recursos formativos y a sus recursos económicos familiares, pero de que sí representan a un gran colectivo que marcará las pautas de las nuevas relaciones laborales.

\subsection{En cuanto a la relación formación/empleo: desequilibrio entre esfuerzos y recompensas}

Las personas con formación superior se encuentran en una situación paradójica, al menos en el contexto laboral español: mientras que su formación y cualificación se supone vital para su inserción profesional y para conseguir determinados puestos de trabajo, en demasiados casos, esa cualificación resulta inútil para llevar a cabo muchos de los empleos en los que se ocupan. El efecto negativo más evidente es la sobrecualificación: se pide una cualificación que no se aplica en el empleo.

Ante esta circunstancia, se observan principalmente dos tipos de comportamientos: uno que consiste en la prolongación de los estudios y la búsqueda de títulos superiores y de mayor especialización que permitan mejorar la posición en el mercado de trabajo:

Cuando terminé Relaciones Laborales, como veía que no tenía curro, pues a la especialización, tienes que hacer algo, algo diferente, o especializarte en lo que sea, porque sólo con la carrera, pues no... no te sirve [...]. Bueno, primero intenté montar una empresa con un amigo y tal, pero no salió. Entonces hice el máster de Medio Ambiente. La idea del máster fue porque nos dijeron que teníamos muchas probabilidades de encontrar trabajo en ese ámbito. Entonces lo hicimos con esa idea. La pena es que no ha sido así, las posibilidades no eran tan claras (28 años, diplomado en Relaciones Laborales, en prácticas).

Pero también se observa otro comportamiento, más difícil de evaluar, que consiste en aspirar a estabilizar una inserción profesional y ocupacional independientemente del título obtenido:

Luego te da rabia, cuando oyes a gente hablar, de tu edad o de más edad, que te dice: "¡Hay que moverse!, ¡el que se mueve, lo consigue!”. Pues mira..., ¡no! Y a mí, que no me vendan milongas. Sí, si tienes suerte. Hay gente que se mueve mucho y no..., y acaba aceptando cosas que..., que no tenía en la cabeza, ni en mente, y al final, te acaba gustando lo que haces y no haces lo que te gusta ( 28 años, licenciado en Bellas Artes, desempleado).

En otras palabras, ante la falta de oportunidades laborales, hay quienes intentan buscar un distintivo superior con el fin de poder mantener sus pretensiones laborales, mientras que hay quienes admiten la depreciación de su título y no tienen más remedio que rebajar sus pretensiones.

Quienes gozan de un mayor nivel educativo se hallan en mejor situación de superar los avatares de la precariedad laboral, tienen más posibilidades de estabilizar su situación laboral y mayores facilidades para la empleabilidad 4 ; sin embargo, la precariedad resulta difícil de sobrellevar cuando se han generado unas expectativas laborales que se niegan y no se consiguen cumplir. Esas expectativas que se generaron durante los estudios universitarios tampoco es que fuesen muy elevadas, ya que, conscientes de la situación laboral, las expectativas suelen limitarse a la búsqueda de 'un trabajo de lo mío', que, sin embargo, resulta ser lo complicado de conseguir. Por esa razón, es durante el periodo de inserción laboral cuando los jóvenes no tienen más remedio que ir transformando y moldeando sus expectativas laborales, atendiendo tanto a las oportunidades como a las limitaciones que les van surgiendo.

\subsection{Sobre la experiencia de los márgenes del empleo: indefinición y precariedad}

Los márgenes del empleo suponen un quicio, una tierra de nadie, un espacio normalmente de tránsito que admite alterar posiciones entre lo formal y lo informal:

Nunca me ha gustado depender de la gente. Entonces empecé a currar, claro, curros... currillos de poca monta, que te dejan seguir estudiando y tal. Pues en un bar los fines de semana, cuidando

${ }^{4}$ Los títulos son cada vez más una condición necesaria para hacer frente a la precariedad laboral, y cada vez menos una condición suficiente para superarla y acceder a puestos de trabajo protegidos. 
un crío y así. Luego, cuando terminé la carrera, ya con la licenciatura, pues me he buscado cosas en relación a lo mío, pero sólo me han salido trabajillos sueltos, proyectos cortos en los que he participado y ahora la beca con la que estoy ( 27 años, licenciada en Física, becaria de colaboración).

En los márgenes, las situaciones laborales son borrosas, quienes las habitan o transitan se mueven entre empleos temporales, entre empleo y desempleo, entre periodos de estudio y de trabajo: esto es, se mueven en una indefinición laboral. La indefinición laboral genera desprotección, precisamente, por no tener cabida en las modalidades habituales de la representación del sujeto trabajador, que son las que están realmente protegidas. Además, genera situaciones en las que es necesario plantearse más que nunca la distinción entre lo que es un empleo y lo que es un trabajo, precisamente, por esa indefinición, en la que se puede estar trabajando sin tener un empleo, como quienes tienen una beca, están en formación y en prácticas o realizan trabajos voluntarios. Así lo expresa este chico becario en el momento de la entrevista:

Vi como la posibilidad más real de trabajar en lo mío. Aunque fuera por un periodo limitado y mediante beca. [...] Poder trabajar en un proyecto personal y en algo que me gustaba. [...] ¡Claro que es un trabajo! A mí me pagan por hacer un trabajo. Lo que pasa que no es un trabajo en regla, sino que es un trabajo más protegido. Y en ese sentido, ya lo marcan, sólo durará cuatro años. [...] Pero yo lo aprovecho como un trabajo más, luego ya se verá (29 años, licenciado en Sociología, becario predoctoral).

Pero esta indefinición laboral en la que podemos encontrar rasgos de precariedad laboral no necesariamente lleva a una precariedad social: la ecuación 'estar' en precario (tener un empleo precario o incluso no tener empleo) igual a 'ser' precario puede llegar a tener demasiadas excepciones. Trabajar en los márgenes del empleo revela, en algunos casos, un recorrido acumulativo que juega como una suerte de socialización laboral, independientemente de cuáles sean las condiciones en las que se realiza la actividad, y más bien ligada al contenido del trabajo y a la valoración subjetiva de éste. Así, puede llegar a ser una experiencia en la que se acumulan saberes y se aprenden maneras y actitudes que posteriormente pueden ser aprovechadas. En efecto, mientras que la precariedad laboral es un fenómeno generalizable a la mayoría de la juventud, para algunas personas jóvenes esa precariedad resultará un tránsito hacia mejores situaciones laborales, como apuntan las trayectorias flexibles, mientras que para otros puede transformarse en una condición permanente de relación con el mercado de trabajo, como sucede en las trayectorias precarizadas.

Sin embargo, en cualquier caso, la necesidad de protección en los márgenes del empleo es vital y determinante también en la proyección de las trayectorias laborales. La experiencia de la precariedad laboral depende de otros factores como el capital social, que fundamentalmente, lo forman la familia y las redes sociales, que funcionan como auténticas “mallas de seguridad” (Moreno Fernández, 2001) y hacen soportable ciertos niveles de precariedad. La protección en estos márgenes del empleo proviene principalmente de esas fuentes, y no del Estado ni de las administraciones locales, cuyas políticas desatienden injustamente aquello que se encuentra entre el empleo y el no empleo. En este sentido, las políticas sociolaborales y los dispositivos de protección deberían atender a las diferentes realidades del trabajo y superar la separación entre quienes tienen empleo formal que les protege y quienes no lo tienen y derivan en sujetos de medidas asistenciales.

\subsection{Sobre las desigualdades de género}

La precariedad laboral viene a reforzar relaciones de género desiguales (Maruani, Rogerat y Torns, 2004). A pesar de que las mujeres, y sobre todo las jóvenes, se forman más y su presencia en el empleo es cada vez mayor, las relaciones de género siguen marcadas por una división sexual del trabajo apenas modificada en lo substancial (Torns, 2005 y 2007).

Ante la prolongación de los años de estudios y las experiencias de precariedad laboral en el acceso al empleo, el futuro familiar de las parejas jóvenes se viene retrasando. La falta de estabilidad económica es una de las principales razones de este retraso, pero en los relatos de las personas jóvenes se aprecia que tampoco hay prisa para ello. En el caso de las mujeres, a esta falta de apremio hacia la maternidad se suma la certeza de las incompatibilidades y limitaciones que les impondrá cuando ésta llegue, sobre todo, si la precariedad laboral se mantiene en sus trayectorias.

Las situaciones de precariedad laboral y de desempleo tornan más problemática aún la ya de por sí difícil conciliación de la vida laboral y familiar. Contrariamente a sus pares masculinos, las jóvenes dejan constancia en sus relatos de que el trabajo doméstico y de cuidados tomará un lugar creciente en sus actividades cotidianas en determinados momentos de la vida y son conscientes de que será complicado mantener trayectorias laborales en continua progresión, y en algunos casos, incluso les será difícil mantener el empleo.

Como hemos comprobado, estas diferencias entre las trayectorias de las y los jóvenes son fruto de las desigualdades que sufren las mujeres en el mercado laboral, ya que, para ellas, la precariedad laboral -principalmente, contractual y salarial- es mayor, y también tienen más posibilidades de que se perpetúe en sus trayectorias. Una precariedad mantenida, como sucede en las trayectorias precarizadas, será determinante en una reflexión como la siguiente:

Tampoco es que quiera tener un niño ya, es algo que lo veo lejano todavía, pero lo que sí me planteo es que, si continúo en esta situación y no 
encuentro nada mínimamente decente, cuando tenga un crío no voy a estar rompiéndome los cuernos por un trabajo sin futuro. Lo dejo y punto (30 años, licenciada en Biología, teleoperadora).

En este sentido, podemos decir que la precariedad y la inestabilidad laboral se encargan de mantener la tradicional división sexual del trabajo, sobre todo cuando las condiciones de precariedad se mantienen a lo largo del tiempo en las trayectorias laborales.

\section{Políticas de empleo y nuevos dispositivos de inserción laboral: de la inserción a la empleabilidad}

A lo largo de las últimas décadas, se ha ido extendiendo e institucionalizando un espacio-tiempo vital entre la formación y el empleo que confiere a quien lo habita estatus muy diversos -estudiantes trabajando, trabajadores estudiando, desempleados en procesos de reciclaje-. Como hemos comprobado a partir de las trayectorias anteriormente expuestas, durante el acceso al empleo no se producen ni integraciones ni exclusiones laborales plenas, lo que esboza un nuevo orden en los sistemas de inserción sociolaboral. Un nuevo orden en el que ya no sirve colocarse laboralmente, buscar una posición en el mercado de trabajo, sino buscarse la vida, que remite a montárselo, es decir, a trabajarse y hacerse un hueco en el mercado laboral (Santamaría, 2010: 113).

Es precisamente en ese acceso al empleo donde se están poniendo en marcha los dispositivos de orientación e intermediación laboral, que se desarrollan a partir de las políticas activas de empleo. Las políticas de fomento del empleo de las personas jóvenes se incluyen entre las políticas activas y combinan medidas de diverso tipo: incentivos a la contratación, programas de formación y reforzamiento de los servicios de orientación, intermediación y colocación, entre otras. Hoy día, las políticas activas de empleo constituyen el referente de lo que los diferentes países europeos deben hacer en materia de bienestar social en relación con la lucha contra el paro y la exclusión social; es en este sentido que se puede hablar de una europeización de las políticas de empleo. De hecho, el enfoque adoptado por las políticas de empleo actuales se encuadra bajo la Estrategia Europea de Empleo, que define cuatro pilares de actuación: innovación y 'espíritu empresarial', adaptabilidad, empleabilidad e igualdad de oportunidades.

Aunque existe una delimitación de los colectivos a los que se dirigen estas políticas, los programas destinados a las personas jóvenes han cobrado especial relevancia en los últimos años y, sobre todo, aquellos orientados al fomento de su empleabilidad. La empleabilidad, desde las instituciones públicas, consiste en fomentar la mejora de las condiciones individuales de las personas para que puedan acceder al mercado de trabajo en igualdad de oportunidades. Debido a que está adquiriendo un interés prioritario en la agenda de las políticas de empleo, se están incrementando los recursos y servicios que buscan mejorar la empleabilidad de la población joven, promoviendo una presencia creciente de agentes de inserción, sean éstos públicos, como los servicios públicos de empleo, o privados, como las empresas de trabajo temporal. Pero aunque se aprecia que la inserción laboral capta cada vez mayor interés político, los recursos siguen siendo insuficientes y no se le otorga importancia real a las consecuencias de la intervención en este campo.

Pero ¿en qué consiste la empleabilidad de las personas jóvenes? Tal y como se presenta en los diferentes documentos que dan forma a la Estrategia Europea de Empleo, se trata de un conjunto de adquisiciones de tipo formativo y curricular, y otras de tipo personal y actitudinal (saber tomar decisiones, saber comportarse, saber venderse en el mercado), que los individuos deben lograr para integrarse en el mercado de trabajo.

Como no se puede asegurar la inserción laboral de las personas jóvenes, ya que requeriría intervenir sobre el mercado laboral y sobre la oferta de empleo, se trabaja sobre la empleabilidad de éstas, convirtiendo, de este modo, el 'hacerse empleable' en una necesidad ya no sólo para acceder al empleo, sino también para mantener un empleo. Y este hecho es el que supone un verdadero cambio en el paradigma de la inserción.

Entre las causas de este cambio en el paradigma de la inserción que favorece la lógica de la empleabilidad, encontramos las siguientes. La principal es la que tiene que ver con la evolución de las nuevas formas de empleo ligadas a la economía de la información y del conocimiento, y que se encuentran mediadas por el desarrollo acelerado de las tecnologías y las nuevas posibilidades que éstas permiten a la hora de realizar y organizar los trabajos. Estas nuevas formas de empleo requieren de personas trabajadoras que se adapten al cambiante mundo productivo y de servicios cada vez más competitivo y a una escala global.

Otra causa, enlazada a la anterior, tiene que ver con la devaluación considerable de la cualificación entendida en su sentido tradicional, es decir, como saberes adquiridos que se aplican en el lugar de trabajo. Actualmente se valora otro tipo de cualificación más difusa, que tiene que ver con competencias personales $y$ actitudinales producto de un saber estar, un saber comunicar, un saber relacionarse. Se demanda tener iniciativa, capacidad de cambio o disponibilidad, cuestiones que tienen que ver con actitudes personales $y$ no con destrezas profesionales. Esto deriva hacia una forma de capacitación en términos de competencias (Alaluf, 2003; Gautié, 2004) que normalmente no son proporcionadas por una cualificación profesional, pero que, sin embargo, se exige que sean puestas a trabajar, es decir, activadas para el empleo.

La empleabilidad se centra en una formación que enfatiza el aprendizaje de competencias personales. 
Se piden habilidades asociadas a conceptos transversales, como la comunicación, la capacidad de diálogo, la capacidad de negociación, el pensamiento asertivo, y la facilidad para plantear y resolver problemas. Y además estas competencias deben ser polivalentes y siempre actualizadas, como si de conocimientos técnicos se trataran. En consecuencia, 'hacerse empleable' se convierte en un trabajo en sí mismo para quien quiera tener o mantener un empleo.

'Hacerse empleable' no sólo constituye, en la actualidad, el ámbito de intervención de las instituciones de empleo, también se ha establecido como un auténtico requisito laboral en boca de sus protagonistas:

Termino la carrera y no me sirve para nada, porque en realidad... si no tienes nada más... Y empiezas... ¿cómo busco y qué es lo que buscas? Al final no sabes, estás un poco desorientado y te tienes que buscar la vida. [...] Hice un curso de inglés financiero y me apunté en X [una empresa de trabajo temporal] (27 años, licenciada en Derecho, temporal por ETT).

Para mí, buscar trabajo es un trabajo en sí mismo. $\mathrm{Y}$ te tienes que organizar la mente muy bien. Saber lo que quieres y, en mi caso, no sabiendo exactamente lo que quiero, bueno, sabiendo qué cosas me gustan, ir desechando las cosas que no, las que no estoy dispuesto a hacer prácticamente bajo ninguna condición, claro (28 años, licenciado en Bellas Artes, desempleado).

Estas prácticas orientadas a la búsqueda de una mayor empleabilidad, el reciclaje profesional, la mejora de las competencias, la asunción de riesgos y la movilización de recursos funcionan como un imperativo personal. Ser una persona emprendedora y potenciar características como la proactividad, la creatividad o la asertividad se traduce en un trabajo que se ejerce sobre sí mismo. Como observa Lorey (2006) analizando el trabajo de productores y productoras culturales, este trabajo sobre uno mismo lleva al foucaultiano gobierno de sí, a la "empresarización de sí”.

La empleabilidad se vuelve aún más persuasiva, viene a significar que cada uno debe 'ocuparse en el esfuerzo de ocuparse', es decir, que al deseo de trabajar, hay que sumarle la motivación para buscar empleo, la capacidad de saber buscarlo, la disposición para adquirir la información necesaria, la capacidad de esfuerzo para mejorar las competencias como trabajador y, además, tener una actitud positiva, ánimo, autodisciplina, afán de superación, confianza en uno mismo, entre otras cuestiones.

La lógica de la empleabilidad no tiene, por tanto, únicamente un calado económico, sino también una profunda ideología política de consecuencias evidentes. Entre ellas, un avanzado proceso de individualización de las personas trabajadoras en el mercado laboral, ya que 'hacerse empleable' se supone una responsabilidad individual que recae principalmente en la persona que quiere acceder o mantener un empleo, y pone el énfasis en que cada persona tiene que hacer frente a su propio destino (Beck, 1998 y 2001; Sennett, 2000 y 2006). Esta apelación a la responsabilidad individual de 'hacerse empleable', presente en los discursos de los y las jóvenes, no sólo en el momento de acceder al empleo, sino también a lo largo de sus trayectorias laborales, hace que los problemas económicos y de funcionamiento del mercado laboral se vean y se vivan como problemas individuales. Se produce lo que algunos investigadores caracterizan como "una psicologización política del trabajo” (Crespo, Revilla y Serrano, 2009). Este proceso consiste básicamente en una transformación resultado de un trabajo de producción política y discursiva por medio del cual los conflictos sociales son transformados en exigencias morales y psicológicas sobre los sujetos. Expresiones como las que siguen reflejan la angustia que generan esas dinámicas individualistas y competitivas:

Ves que todo el mundo está muy preparado, que viene gente joven con cuatrocientas cincuenta cosas y que... tú eres de la promoción anterior y que estás un poco desfasado (27 años, licenciada en Derecho, temporal por ETT).

[...] es que me encuentro ya en un nivel en el que no me puedo reciclar, por ejemplo, para competir con chavales de 22 años... Sí, veo que, con mi edad, para ciertas cosas ya no sirvo [para presentarse a ciertas ofertas de empleo], ya ni pierdo el tiempo (28 años, licenciado en Bellas Artes, desempleado).

Por lo tanto, contrariamente a lo que las instituciones proclaman -efectos redistributivos e igualitarios entre los ciudadanos-, las políticas activas de empleo y la intermediación sociolaboral, bajo la lógica de la empleabilidad, contribuyen a engordar ese espacio de inserción constante y reiterativa al que tienen que hacer frente las personas jóvenes, a través de la exigencia imperiosa, pero al mismo tiempo inabarcable, de 'hacerse empleables'.

\section{Consideraciones finales: la precariedad como referente identitario}

Sosteníamos al principio de este artículo que el escenario flexible y precario de lo sociolaboral funciona como sustrato de la nueva economía de mercado, en la cual de poco sirven aquellos modelos de la modernidad con base en los cuales se podía pensar la estructura laboral, con la existencia de un centro y una periferia claros, y una movilidad social, bien ascendente bien descendente, pero siempre en el surco de una línea definida en biografías estandarizadas. A lo largo de la investigación, hemos podido comprobar que las situaciones laborales de las personas jóvenes y las biografías que fomentan entre ellas tienen que ser pensadas como un orden complejo, escurridizo en muchos casos, y repleto de conexiones no siempre previstas de antemano, 
en el cual ya no sirve ni la división centro/periferia ni tampoco los análisis en términos de ganadores y perdedores (Fenton y Dermott, 2006).

En este contexto de complejidad, incertidumbre y de crisis de la ciudadanía laboral (Alonso, 2007), se hace necesario reflexionar sobre lo que significan las diversas maneras de convertirse en trabajador o trabajadora actualmente, pero, sobre todo, convertirse en trabajadores y trabajadoras 'de otro tipo'. Desde que el trabajo cambió sus formas, ser 'sujeto trabajador' ha dejado de ser, para muchas personas, un dato objetivo y se ha convertido en un trabajo subjetivo. Este trabajo es el que tienen que llevar a cabo quienes acceden por primera vez al empleo, pero también el de quienes tras perder, dejar o terminar un empleo comienzan la búsqueda de otro, o el de quienes buscan una alternativa a su empleo actual. Todas estas personas se verán inmersas en el proceso de lo que, páginas atrás, hemos descrito como 'hacerse empleable'. El 'ser trabajador' que antes venía dado, principalmente, por la dedicación al empleo, pero también por la edad, la formación o la vocación, ha pasado, actualmente, a ser un trabajo en sí mismo. No sólo porque, cada vez más, los empleos otorgan la categoría de trabajador únicamente de forma temporal, sino también porque conseguir un empleo y mantenerlo es un trabajo en sí mismo.

En el análisis léxico de las entrevistas, hemos podido comprobar cómo se manejan mucho menos las formas intransitivas como el 'ser' y el ‘estar', más acordes con la norma de empleo moderno, que las transitivas como el 'tener' y el 'hacer', que encajan mejor con las nuevas dinámicas económicas. Éstas son claves para comprender los procesos de construcción identitaria contemporáneos, en los que la precariedad surge como referente de las nuevas subjetividades.

Resulta interesante subrayar cómo las políticas públicas de empleo recogen y alimentan estos cambios, poniendo el énfasis en la empleabilidad. Es importante conocer cuál es el discurso con el que se manejan las políticas de empleo y el papel que cumplen los agentes que las aplican, identificando las consecuencias y efectos que ejercen sobre las personas trabajadoras. Porque, como hemos podido verificar en esta investigación, su papel de (inter)mediadores está cada vez más presente y está teniendo una clara influencia sobre el imaginario laboral, sobre la producción de sentidos en torno al empleo y también sobre la subjetividades laborales.

En resumen, la individualización y la psicologización en la forma de tratar la inserción laboral juvenil está alimentando un modo de subjetivación que se adecua perfectamente al actual desarrollo económico del capitalismo avanzado y a la debilidad de unos Estados de bienestar que, ante la imposibilidad de generar empleo y de regularlo de forma que proteja a las personas trabajadoras de la inestabilidad e inseguridad económica, se encarga de la empleabilidad de los individuos. 


\section{Referencias bibliográficas}

ALALUF, M. (2003): “La sociología del trabajo a través de una relectura de Claude Durand: situación de trabajo, cualificaciones, competencias y clase obrera", en Revista de Relaciones Laborales, nํㅡ 2, págs. 93-102.

ALONSO, L. E. (2007): La crisis de la ciudadanía laboral, Barcelona, Anthropos.

BECK, U. (2001): "Vivir nuestra propia vida en un mundo desbocado: individualización, globalización y política”, en GIDDENS, A.; y HUTTON, W. (eds.), En el límite. La vida en el capitalismo global, Barcelona, Tusquest, págs. 233-245.

- (1998) [1992], La sociedad del riesgo: hacia una nueva modernidad, Barcelona, Paidós.

CASAL, J. (1996): “Modos emergentes de transición a la vida adulta en el umbral del siglo XXI: aproximación sucesiva, precariedad y desestructuración", Revista Española de Investigaciones Sociológicas, ํㅜㄱㅜ págs. 295-316.

CASAL, J. et al. (2006): “Aportaciones teóricas y metodológicas a la sociología de la juventud desde la perspectiva de la transición”, Papers, no79, págs. 21-48.

CRESPO, E., REVILLA, J. C.; y SERRANO, A., (2009), "Del gobierno del trabajo al gobierno de las voluntades: el caso de la activación", Psicoperspectivas, vol. VIII, $\mathrm{n}^{\mathbf{0}} \mathbf{2}$, págs. 83-101.

DAVIA, Ma A. (2004): La inserción laboral de los jóvenes en la Unión Europea. Un estudio comparativo de trayectorias laborales, Madrid, Consejo Económico y Social.

DU BOIS-REYMOND, M.; y LÓPEZ BLASCO, A. (2004): "Transiciones tipo yo-yo y trayectorias fallidas: hacia las políticas integradas de transición para los jóvenes europeos”, Revista de Estudios de Juventud, n으. 65, págs. 11-29.

EUROPEAN GROUP FOR INTEGRATED SOCIAL RESEARCH (2001): "Misleading trajectories: Transition dilemmas of young adults in Europe", Journal of Youth Studies, vol. 4, ํㅡ 1. págs. 101-118.

FENTON, S.; y DERMOTT, E. (2006): “Fragmented careers? Winners and losers in young adult labour markets", Work, Employment and Society, vol. $20, \mathrm{n}$ 을 2, págs. 205-221.

GARCÍA-MONTALVO, J.; y PEIRÓ, J. Ma (2001): Capital humano, el mercado laboral de los jóvenes: formación, transición y empleo, Valencia, Fundación Bancaja.

GARCÍA BLANCO, J. M.; y GUTIÉRREZ, R. (1996): “Inserción laboral y desigualdad en el mercado de trabajo: cuestiones teóricas”, Revista Española de Investigaciones Sociológicas, $\mathrm{n}-75$, págs. 269-293.

GARCÍA ESPEJO, I. (1998): Recursos formativos e inserción laboral de jóvenes, Madrid, CIS; Siglo XXI.

GASH, V. (2008): “Bridge or trap? Temporary workers' transitions to unemployment and to the standard employment contract", European Sociological Review, vol. 24, $\mathrm{n}^{0} 5$, págs. 651-668.

GAUTIÉ, J. (2004): “Repensar la articulación entre mercado de trabajo y protección social en el postfordismo", Cuadernos de Relaciones Laborales, vol. 22, nํㅜ 1, págs. 147-184.

LOREY, I. (2006): “Gubernamentalidad y precarización de sí. Sobre la normalización de los productores y productoras culturales", Transversal, enero [〈http://transform.eipcp.net/transversal/1106/ lorey/es>]. 
MACHADO PAÍS, J. (2007): Chollos, chapuzas, changas. Jóvenes, trabajo precario y futuro, Barcelona, Anthropos; Azcapotzalco, Universidad Autónoma de México.

MARTÍN CRIADO, E. (1998): Producir la juventud, Madrid, Istmo.

MARTÍNEZ MARTÍN, R. (2003): “La inserción laboral de los universitarios a través de las prácticas en empresas", Revista Española de Investigaciones Sociológicas, no 101, págs. 229-254.

- (2000): “Aproximaciones teóricas a los procesos de inserción laboral”, Revista del Ministerio de Trabajo y Asuntos Sociales, no-26, págs. 65-91.

MARUANI, M.; ROGERAT, C.; y TORNS, T. (dirs.) [2004]: Las nuevas fronteras de la desigualdad. Hombres y mujeres en el mercado de trabajo, Barcelona, Icaria.

MORENO FERNÁNDEZ, L. (2001): “Estados del Bienestar y 'mallas de seguridad'", en MORENO FERNÁNDEZ, L. (ed.), Pobreza y exclusión: la 'malla de seguridad' en España, Madrid, CSIC, págs. 17-50.

SANTAMARÍA, E. (2011): Trayectorias laborales en los márgenes del empleo: políticas, subjetividades y experiencias de jóvenes en la precariedad laboral, serie Premio Realidad Social Vasca, Vitoria-Gasteiz, Servicio Central de Publicaciones del Gobierno Vasco.

- (2010): “'Buscarse la vida’: trayectorias y experiencias de precariedad en el acceso al empleo de las personas jóvenes", Revista de Estudios de Juventud, no 89, págs. 101-123.
SANTOS ORTEGA, J. A.; y SERRANO PASCUAL A. (2006): "El giro copernicano del desempleo actual", Cuadernos de Relaciones Laborales, vol. 24, no 2, págs. 1-19.

SENNETT, R. (2006): La cultura del nuevo capitalismo, Barcelona, Anagrama.

- (2000): La corrosión del carácter. Las consecuencias personales del trabajo en el nuevo capitalismo, Barcelona, Anagrama.

TOHARIA, L.; y CEBRIÁN, I. (2007): La temporalidad en el empleo: atrapamiento y trayectorias, Madrid, Ministerio de Trabajo y Asuntos Sociales.

TORNS, T. (2007): “El tiempo de trabajo y las relaciones de género: las dificultades de un cambio ineludible", en PRIETO, C. (ed.), Trabajo, género y tiempo social, Madrid, Universidad Complutense; Editorial Hacer, págs. 269-278.

- (2005): "De la imposible conciliación a los permanentes malos arreglos", en Cuadernos de Relaciones Laborales, vol. 23, ํㅜ 1.

VERD, J. M.; y LÓPEZ ANDREU, M. (2012): “La inestabilidad del empleo en las trayectorias laborales: un análisis cuantitativo", Revista Española de Investigaciones Sociológicas, $\mathrm{n}-138$, págs. $135-148$

WALTHER, A. (2006): "Regimes of youth transitions. Choice, flexibility and security in young people's experiences across different European contexts", Young. Nordic Journal of Youth Research, vol. 14, no 2, págs. 119-139. 